\title{
Chaotic analysis of seismic time series and short term forecasting using neural networks.
}

\author{
V.P. Plagianakos and E. Tzanaki \\ Department of Mathematics, \\ University of Patras, \\ University of Patras Artificial Intelligence Research Center (UPAIRC). \\ GR-261.10 Patras, Greece. \\ e-mail: $\{v p p$, helen\}@math.upatras.gr
}

\begin{abstract}
In this study, a chaotic analysis approach was applied to a time series composed of seismic events occurred in Greece. The dynamics of the earthquakes belong to the category of dissipative systems, which exhibit chaotic behavior. After the chaotic analysis, short term forecasting using an Artificial Neural Network has been performed. Neural networks, under appropriate conditions, are known to be universal function approximators, thus they have been investigated as tools for time series forecasting. Here, a neural network is trained to make short term earthquake predictions. The network architecture has been dictated by the calculated characteristics of the time series itself. Preliminary results indicate that this is a promising approach.
\end{abstract}

\section{Introduction}

One of the central problems of science is forecasting. Given the past, how can we predict the future. If we have a system that doesn't change with time then it is very easy to predict the next value. We take the last observation as a forecast for the next one. If the system changes periodically with time all we have to do is to observe a full cycle. On the other it is very difficult to make a prediction for random numbers. The systems that we are interested in are something in between. They are not periodic but they contain some kind of structure, which can be exploited to obtain better predictions. If the predictions are perfect, then the system is completely deterministic. If the predictions are good, but not perfect, then the system has a deterministic component. If the predictions are terrible, then the system is not deterministic at all [4].

Starting with a sequence of measurements, $x\left(t_{i}\right)$, which formulate the time series, our purpose is to determine what the data themselves can reveal about the dynamics. In particular, we will discuss some tools from time series analysis that can sometimes be used to suggest what types of equations are appropriate, or to compare the predictions made by mathematical models to measurements made in the field. The ultimate goal for time-series analysis might be to construct a computer program that, without any knowledge of the physical system from which the data come, can take the measured data as input and provide as output a mathematical model describing the data [5].

The rest of the paper is organized as follows: the next Section, describes the fundamentals of chaotic time series analysis theory. In Section 3 the proposed methodology is introduced, while in Section 4, the neural network architecture employed is highlighted. Section 5 concludes the paper.

\section{Chaotic Time Series Analysis}

When dealing with data, we need to introduce two new concepts: measurement and noise. In conducting an experiment we can measure only a limited set of quantities and we are able to make those measurements with limited precision. Measurement noise refers to fluctuations in measurements that arise from chance. Dynamical noise is another important source of noise in data. Real-world systems do not exist in isolation; they are affected by outside influences [5].

Since there is neither dynamical nor measurement noise, the model is completely deterministic. This means that, in principle, if we have the initial condition we can accurately calculate the future values. One the other hand, if the model is chaotic there may be practical limitations on our ability to predict it. Most techniques for nonlinear data analysis involve two steps:

1. In the first step, the data are used to reconstruct the dynamics of the system.

2. The second step involves characterization of the reconstructed dynamics.

More generally (for the first step), the time series is embedded in an m-dimensional space, by taking $\mathrm{m}$ consecutive data points, $x\left(t_{i}\right)$ to define the reconstructed time series: 


$$
\left.R\left(t_{i}\right)=\left\{x\left(t_{i}\right), x\left(t_{i}+\tau\right), \ldots, x\left(t_{i}+(m-1) \tau\right)\right)\right\} .
$$

This technique of representing a measured time series as a sequence of points in an m-dimensional space is called time lag embedding. There is an important theorem [10] (Taken's embedding theorem), which states that the reconstructed dynamics are geometrically similar to the original for both continues-time and discrete-time series. The sequence of points created by embedding a time series is called the trajectory of the time series, $m$ is called the embedding dimension, and $\tau$ is the embedding lag or delay time [5]. Our purpose is to find an optimal value for the embedding dimension, in order to use this information in the neural network architecture.

The second step is the characterization of the time series. To this end, we have to evaluate some fundamental quantities, such as the correlation integral and dimension, and the maximum Lyapunov exponent.

When the trajectories of a dynamical system diverge over the course of time and this separation is exponentially fast, then chaos exists. The properly averaged exponent of this increase is characteristic for the system underlying the data and quantifies the strength of chaos. It is called the maximum Lyapunov exponent. More generally the Lyapunov exponents shows us the sensitive dependence on initial condition [4].

The correlation dimension is one of the most important quantities in chaotic time series analysis. Dissipative dynamical systems that exhibit chaotic behavior often have an attractor in phase space, which is strange. Strange attractors are typically characterized by a fractal dimension, $\mathrm{D}_{0}$, which is very difficult to compute (whenever $\mathrm{D}>2$ ). But there is another measure of the dynamical system; the so-called correlation dimension, $v$, which can be relatively easy to obtain from the time series.

Let us define the correlation integral in some vector space to be the fraction of all possible pairs of points which are closer than a given distance $r$, in a particular norm:

$$
C(r)=\lim _{N \rightarrow \infty} \frac{1}{N^{2}} \sum_{i, j=1}^{N} \theta\left(r-\left\|\vec{X}_{i}-\vec{X}_{j}\right\|\right),
$$

where $\theta(\mathrm{x})$ is the Heaviside step function.

The most important attribute of correlation integral is that behaves as a power of $r$ :

$$
\mathrm{C}(\mathrm{r}) \approx \mathrm{r}^{v} \text {. }
$$

The exponent $v$ is called correlation dimension and is closely related to the fractal dimension [1].

\section{The proposed methodology}

Now that we have reviewed the theory, let us put it into practice and consider a time series composed of seismic events recorded in Greece. The dynamic of earthquakes has attracted much attention as a difficult real-world problem.

To apply the aforementioned theory, we have to suppose that earthquake dynamics belong to the category of dissipative systems, which can reveal dynamics with strange attractor.

In the following we use the reconstructed time series:

$$
\left.R\left(t_{i}\right)=\left\{x\left(t_{i}\right), x\left(t_{i}+\tau\right), \ldots, x\left(t_{i}+(m-1) \tau\right)\right)\right\},
$$

where $\tau$ is a delay time and $m$ is the embedding dimension. The embedding dimension has found to be 5. In order to estimate $\mathrm{m}$, several trials were conducted, using consecutive values for $\mathrm{m}$, until the underlying attractor has found to have structure. This is a difficult trial-and-error process driven by experience.

The correlation dimension $v$ of the reconstructed time series is given as:

$$
v=\lim _{r \rightarrow \infty, m \rightarrow \infty} \frac{d \ln C(r, m)}{d \ln (r)} .
$$

To estimate the maximum Lyapunov exponent, $\lambda$, which is a measure to estimate the strength of chaos, the following relation is used:

$$
\delta_{n} \approx \delta_{0} e^{\lambda n \Delta t} \Rightarrow \lambda=\lim _{n \rightarrow \infty} \frac{\log \left|\delta_{n} / \delta_{0}\right|}{n \Delta t},
$$

where $\delta_{\mathrm{n}}$ is the divergence of the trajectories over the time [7].

In Figure 1, the seismic events (with magnitude greater than 2 degrees of Richter scale) that occurred in Greece the last twenty years are exhibited.

To better analyze the seismic data, we have separated them in four sets, according to the geographical region that the earthquake took place. Here, we present results only from one highly seismic region; Zakynthos island (Figure 2). The results are particularly interesting and (combined with those of the other three regions) give us a better insight to the earthquake dynamics. 


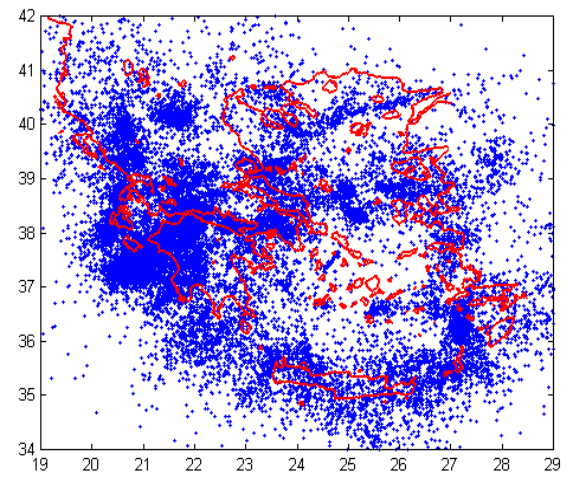

Figure 1: Plot of the seismic events in Greece

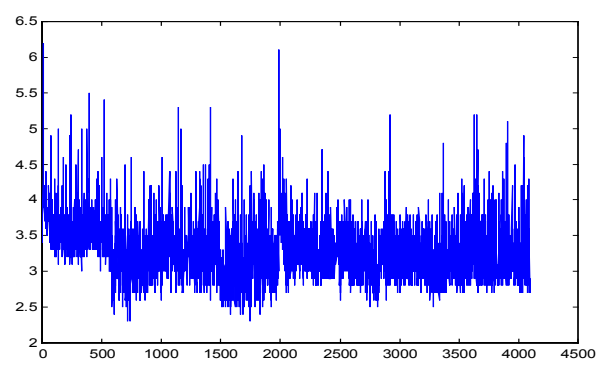

Figure 3: Plot of the magnitude of the earthquake vs. time

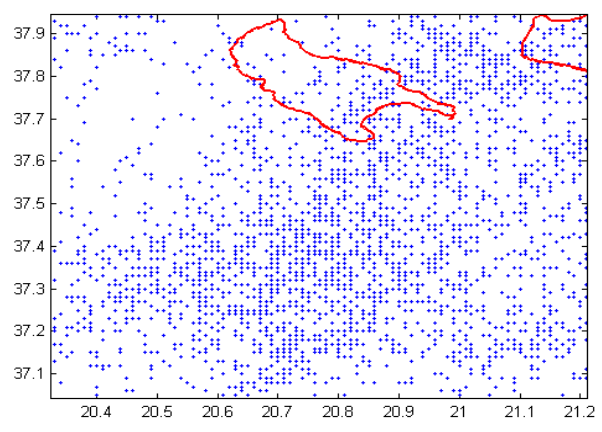

Figure 2: Plot of the seismic events at the Zakynthos island area

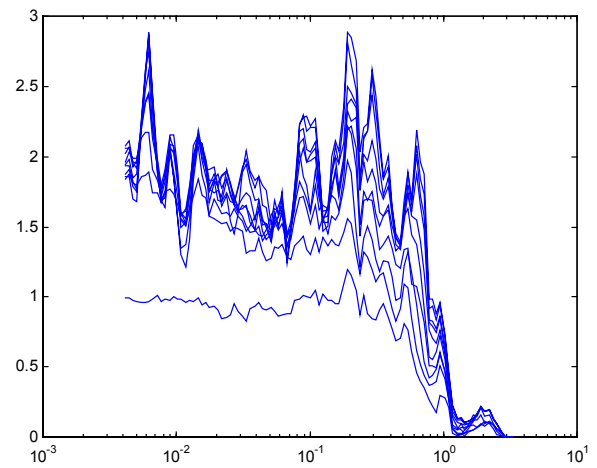

Figure 4: Correlation dimension plot

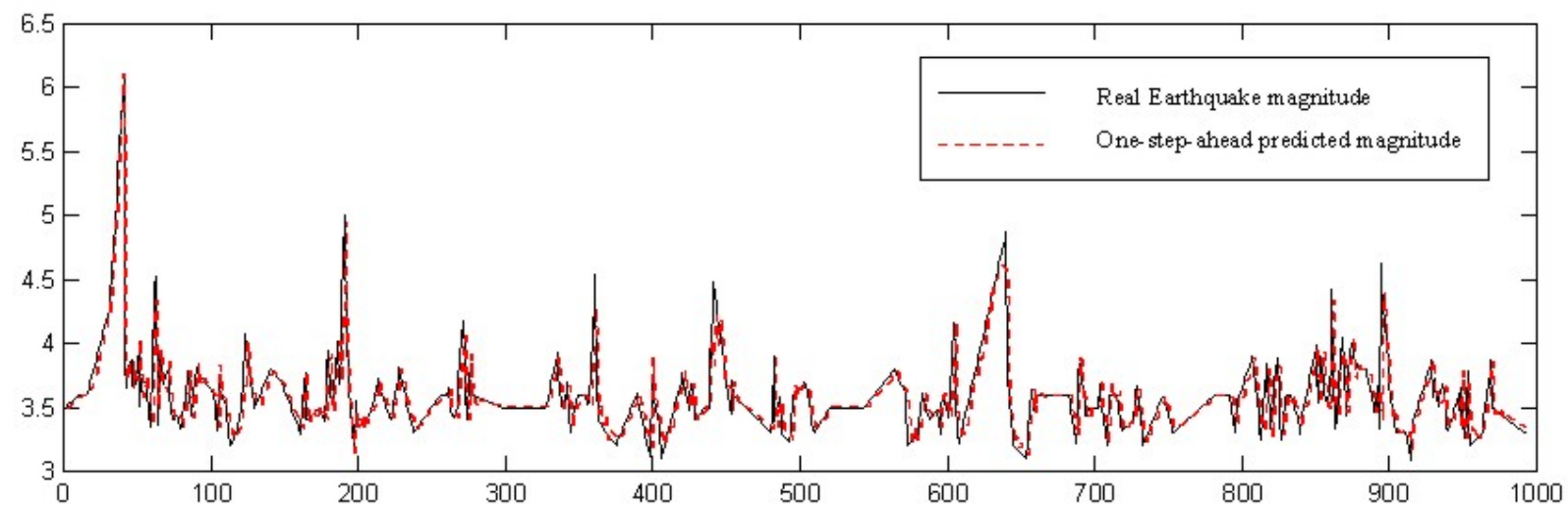

Figure 5: Real and one-step-ahead predicted earthquake magnitude 
The time series for the above mentioned data are shown in the Figure 3. In this Figure, the relation between the magnitude of the earthquake and time is plotted.

Dealing with real data is a difficult task, because one has to be very careful in the selection of the data points. First of all, the data points must be taken in equal time. In other words, for the prediction to have any value the time series must consists of values which are spaced uniformly in time.

Unfortunately, the time series of the earthquakes doesn't meet the above assumption, since seismic activity is not a periodical phenomenon. Therefore, the use of an interpolation technique is necessary. We have experimented with linear and cubic interpolation, and splines. The new data after the linear interpolation tend to be very close to the mean value, thus missing earthquakes with large magnitude. Splines, on the other hand, seem to perform worse than the cubic interpolation, which was actually used in our experiments. Additionally, a noise reduction algorithm [9] has been used.

Then, the correlation dimension of the underlying attractor is estimated, with the help of TISEAN software package for the chaotic analysis of time series [2]. The correlation dimension was estimated to be between 1 and 2.5, as it can be concluded from Figure 4. Finally, using the method described in [3], the maximum Lyapunov exponent was estimated to be 0.3747 .

From the above it is evident that although the positive Lyapunov exponent shows sensitive dependence on the initial conditions of the supposed earthquake dynamics, the dimension of the underlying attractor is low. Since the underlying system is described by low dimensional chaotic dynamics short term predictions are feasible, and worth trying.

\section{Prediction Using Neural Networks}

Applying the above techniques, information about the nature of the underlying system has been gathered and aided the construction of the ANN. Firstly, the low dimensional attractor indicated that predictions were feasible, although, as it is well known, the dynamical system has chaotic behavior.

After the characterization of the time series is performed, the estimated embedding dimension $(m=5)$ has been used as the number of input nodes.
Specifically, a 5-30-1 feed-forward ANN using logistic activation functions has been used.

The aim was to train the ANN to perform short term forecasting. 10,000 consecutive 5-dimensional vectors constitute the training set, while the generalization capability of the network was tested using 1,000 test vectors. The network was trained by a recently proposed learning algorithm [7] to perform one-step-ahead forecast. The magnitude of the earthquake is forecasted one step at the time and the actual rather than the forecasted magnitude is then used for the next prediction in a forecasting horizon. As shown in Figure 1 , the prediction results were satisfactory.

\section{Conclusions}

A chaotic analysis approach was applied to a time series composed of seismic events occurred in Greece the last twenty years. The first step was the reconstruction of the time series in order to convert the observations into space vectors. Then, the embedding dimension has been estimated. Finally, the maximum Lyapunov exponent and the correlation dimension have been calculated. The reason that we estimate the correlation dimension is that we want to ensure that there exists a low dimensional strange attractor behind our data, while the Lyapunov exponent is a measure of the sensitivity to the initial conditions. For the computation of the above-mentioned quantities, the TISEAN software package has been used [2]. With these tools in hand, an ANN trained for onestep-ahead forecast exhibits satisfactory results.

Regarding the seismic time series from all four regions, the correlation dimension of the underlying attractor was estimated to be between 2 and 3 , while the maximum Lyapunov exponent was found to be 0.71 . Thus, the underlying system is described by low dimensional chaotic dynamics. In a future correspondence we intend to present results taking into consideration all four regions, as well as try accurate predictions using already predicted values.

\section{References}

[1] P. Grassberger and I. Procaccia, "Characterization of Strange Attractors", Physical Review Letters, 50, (1983). 
[2] R. Hegger, H. Kantz, and T. Schreiber, "Practical implementation of nonlinear time series methods: The TISEAN package", Chaos, 9, 413, (1999).

[3] H. Kantz, "A robust method to estimate the maximal Lyapunov exponent of a time series", Phys. Lett. A, 185, (1994).

[4] H. Kantz and T. Schreiber, Nonlinear Time Series Analysis, Cambridge University Press, (1997).

[5] D. Kaplan and L. Glass, Understanding Nonliear Dynamics, Springer (1995).

[6] F. Lisi and R.A. Schiavo, "A comparison between neural networks and chaotic models for exchange rate and predictions", Computational Statistics \& Data Analysis, 30, 87-102, (1999).
[7] G.D. Magoulas, V.P. Plagianakos, and M.N. Vrahatis, "Hybrid Methods Using Evolutionary Algorithms for On-line Training", In Proc. of the IEEE Int. Joint Conf. on Neural Networks (IJCNN'2001), (2001).

[8] G.P. Pavlos, L. Karakatsanis, J.B. Latoussakis, D. Dialetis, and G. Papaioannou, "Chaotic Analysis of a Time Series of Seismeic Events Recorded in Japan", Int. Journal of Bifurcation and Chaos, 4, (1994).

[9] T. Schreiber, "Extremely Simple Nonlinear Noise Reduction Method”, Phys. Rev. E , 47, 2401, (1993).

[10] F. Takens, "Detecting Strange Attractors in Turbulence", Lecture Notes in Math., 898, 226-381, Springer, New York, (1981). 